\title{
Perdas na colheita mecanizada da cana-de-açúcar em resposta a diferentes velocidades da colhedora e de rotação do extrator primário
}

\author{
Murilo Fuentes Pelloso ${ }^{1}$, Adriano Aparecido de Lima ${ }^{2}$, Bruno Fuentes Pelloso ${ }^{3}$, Arthur Pereira da Silva ${ }^{4}$ \\ ${ }^{1}$ Universidade Estadual de Maringá - UEM. ${ }^{2}$ Raizen. ${ }^{3}$ Glencane Bioenergia. ${ }^{4}$ lowa State University. E-mail: \\ murilof.pelloso@hotmail.com
}

\section{Resumo}

O presente estudo objetivou avaliar as perdas visíveis e invisíveis na colheita mecanizada da cana-de-açúcar mediante uso de diferentes velocidades de deslocamento da colhedora e de rotação do extrator primário em canavial de primeiro corte, com terreno a 7\% de declividade. 0 ensaio foi conduzido em Junho de 2014, por ocasião da colheita mecanizada em canavial de primeiro corte (cana de ano), com estimativa de produtividade de $75 \mathrm{t} \mathrm{ha}^{-1}$ no município de Pacaembu, São Paulo. O canavial era formado pela variedade RB96-5902, com 12 meses de idade, em espaçamento de 1,4 metros entre linhas. A colheita foi realizada utilizando uma colhedora da marca John Deere, modelo 3520 acompanhada de um trator, acoplado ao transbordo. Os tratamentos avaliados correspondem a combinações de duas velocidades de deslocamento da colhedora $\left(\mathrm{km} \mathrm{h}^{-1}\right)$ e duas velocidades de rotação do extrator primário (rpm), além das velocidades habitualmente utilizadas pela usina, como testemunha: $3 \mathrm{~km} \mathrm{~h}^{-1}$ a $700 \mathrm{rpm}, 3 \mathrm{~km} \mathrm{~h}^{-1}$ a $1200 \mathrm{rpm}, 5 \mathrm{~km} \mathrm{~h}^{-1}$ a $950 \mathrm{rpm}$ (testemunha), $6 \mathrm{~km} \mathrm{~h}^{-1}$ a $700 \mathrm{rpm} \mathrm{e} 6 \mathrm{~km} \mathrm{~h}^{-1}$ a $1200 \mathrm{rpm}$. As Variáveis respostas analisadas foram as perdas nas formas de tocos, despontes, toletes, pedaços, canas inteiras, estilhaços e perdas totais, em $\mathrm{t}$ $\mathrm{ha}^{-1}$ e a porcentagem total de perdas. Foram observadas respostas significativas $(p \leq 0,05)$ para todas as formas de perdas em função dos tratamentos, com exceção para as perdas nas formas de cana inteira e despontes. Concluiu-se que o aumento da rotação do extrator primário e redução na velocidade de locomoção da colhedora tendem a ocasionar aumento nas perdas de cana-de-açúcar, sendo, nas condições do presente estudo, indicada a velocidade de $3 \mathrm{~km} \mathrm{~h}^{-1}$ com rotação do extrator primário de $1200 \mathrm{rpm}$.

Palavras chave: Perdas visíveis; perdas invisíveis; perdas totais; mecanização; Saccharum officinarum.

Losses on the mechanized harvest of sugar cane in response to different harvester speeds and rotation of primary extractor

\begin{abstract}
The present study aimed to evaluate the visible and invisible losses in mechanized harvesting of sugarcane by using different speeds of the harvester's displacement and rotation of the primary extractor in first-cut cane fields, with $7 \%$ slope. The trial was conducted in June 2014, at the time of mechanical harvesting in first-crop cane (cane of year), with a productivity estimate of $75 \mathrm{t} \mathrm{ha}^{-1}$ in the city of Pacaembu, São Paulo. The sugarcane was formed by the variety RB96-5902, with 12 months of age, in spacing of 1.4 meters between rows. Harvesting was performed using a John Deere harvester, model 3520 accompanied by a tractor, coupled to the transhipment. The treatments evaluated correspond to combinations of two speeds of displacement of the harvester $\left(\mathrm{km} \mathrm{h}^{-1}\right)$ and two speeds of rotation of the primary extractor $(\mathrm{rpm})$, in addition to the speeds usually used by the plant as a control: $3 \mathrm{~km} \mathrm{~h}^{-1}$ at $700 \mathrm{rpm}, 3 \mathrm{~km} \mathrm{~h}^{-1}$ at $1200 \mathrm{rpm}, 5$ $\mathrm{km} \mathrm{h}^{-1}$ at $950 \mathrm{rpm}$ (control), $6 \mathrm{~km} \mathrm{~h}^{-1}$ at $700 \mathrm{rpm}$ and $6 \mathrm{~km} \mathrm{~h}^{-1}$ at $1200 \mathrm{rpm}$. The analyzed variables were the losses in the forms of stumps, bluffs, billets, pieces, whole reeds, shards and total losses, in $\mathrm{t} \mathrm{ha}^{-1}$ and the total percentage of losses. Significant responses $(p \leq 0.05)$ were observed for all forms of losses as a function of treatments, except for losses in whole cane and shoot forms. It was concluded that the increase of the rotation of the primary extractor and reduction in the speed of locomotion of the harvester tend to cause an increase in the losses of sugarcane, being, in the conditions of the present study, indicated the speed of $3 \mathrm{~km} \mathrm{~h}^{-1}$ with rotation of the primary extractor of $1200 \mathrm{rpm}$.

Keywords: Visible losses; invisible losses; total losses; mechanization; Saccharum officinarum.
\end{abstract}




\section{Introdução}

A cultura da cana-de-açúcar (Saccharum officinarum L.) é produzida na maioria dos países tropicais e subtropicais, sendo o Brasil o principal produtor e exportador da cultura e seus diversos subprodutos, como o açúcar e etanol (ANTUNES et al. 2017).

A área total ocupada pela cana-deaçúcar no Brasil é de aproximadamente 9 milhões de hectares, sendo o Estado de São Paulo o maior representante nacional, com área aproximada de 5,3 milhões de hectares ocupados pela cultura e com produção que representa $59 \%$ do total produzido no país (CONAB, 2018).

Conforme destacado por Ripoli et al. (2001), a colheita onera elevados custos para o sistema de produção, além de apresentar diversas dificuldades operacionais, independentemente se realizada de forma manual ou mecanizada. Entretanto, diversos aspectos socioeconômicos, legais, ambientais e do próprio desempenho vem induzindo cada vez mais a mecanização do processo em detrimento a colheita manual. Deve-se destacar ainda o fato de uma colhedora mecânica realizar o trabalho equivalente a aproximadamente 100 cortadores manuais (COSTA NETO, 2006).

Apesar das diversas vantagens advindas da colheita mecanizada, sobretudo quanto ao rendimento, há sempre necessidade de maximizar a qualidade do processo, reduzindo custos e perdas ocasionadas durante sua execução. Dentre as características da colheita mecanizada que devem ser consideradas, os índices de perdas durante o processo devem ser reduzidos, sendo possível faze-lo através da utilização mais adequada possível da colhedora.

As perdas ocorridas durante a colheita da cana-de-açúcar podem ser divididas entre perdas visíveis e invisíveis. As perdas denominadas visíveis constituem-se basicamente de tocos, despontes, toletes, pedaços e canas inteiras. Essas perdas podem ser facilmente identificadas visualmente a campo após a colheita e determinadas por coleta manual e pesagem. Já as perdas invisíveis, na forma de caldo, serragem e estilhaços de cana, que ocorrem devido à ação de mecanismos rotativos que cortam, picam e limpam a matéria prima durante $o$ processamento no interior das colhedoras, dificilmente são identificadas visivelmente a campo, sendo que, apesar de sua importância, a difícil quantificação faz com que as mesmas sejam ignoradas ou pouco consideradas por muitos autores, total ou parcialmente (BURLEIGH, 1988; NEVES et al., 2006; MAGALHÃES et al. 2008; APOLINÁRIO et al. 2017).

Dentre as formas de elevar o rendimento da colheita mecanizada da cana-deaçúcar, pode-se citar o aumento da velocidade de locomoção da colhedora e de rotação do extrator primário, entretanto, as maiores velocidades tendem a elevar as perdas e os níveis de impurezas na carga de cana colhida, por conter maior massa a ser processada pela colhedora, havendo necessidade de ajuste das velocidades ideais em função das características do talhão, porte e condições do canavial e produtividade estimada (RIPOLI; RIPOLI, 2004; YOUNGER, 1980; NEVES et al., 2006).

Diante do exposto, o presente estudo objetivou avaliar perdas visíveis e invisíveis na colheita mecanizada da cana-de-açúcar mediante uso de diferentes velocidades de deslocamento da colhedora e de rotação do extrator primário em canavial de primeiro corte com terreno a $7 \%$ de declividade.

\section{Material e Métodos}

O experimento foi conduzido com tratamentos em delineamento inteiramente casualizado no dia 23 de Junho de 2014, por ocasião da colheita mecanizada em canavial de primeiro corte (cana de ano), com estimativa de produtividade de $75 \mathrm{t} \mathrm{ha}^{-1}$, na fazenda Vista Alegre, situada na latitude de $21^{\circ} 23^{\prime} 37^{\prime \prime}$ Sul, longitude $51^{\circ} 15^{\prime} 10^{\prime \prime}$ Oeste, e com altitude aproximada de $425 \mathrm{~m}$, no município de Pacaembu, Oeste do Estado de São Paulo. O clima da região é caracterizado segundo escala de Köppen-Geiger como temperado úmido com Verão quente ( $\mathrm{Cfa})$. A declividade do terreno no talhão colhido para fins de avaliação era de $7 \%$, sendo esta favorável à colheita mecanizada (BRAUNBECK; OLIVEIRA, 2006).

O canavial, colhido mecanicamente na forma de cana crua, era formado pela variedade RB96-5902, com 12 meses de idade, conduzida em espaçamento de 1,4 metros entre linhas. A variedade é considerada precoce e de boa colheitabilidade mecânica, comumente colhida entre os meses de abril e julho. 
A colheita foi realizada utilizando uma colhedora da marca John Deere, modelo 3520, fabricada em 2012, com 342 cavalos de potência e rodado de esteira. A colhedora possuía faquinhas novas e facão médio, com angulação de corte da base de $13^{\circ}$ e altura de corte em torno de 3 a $4 \mathrm{~cm}$. A colhedora operou no canavial acompanhado de um trator John Deere 4×2 TDA (tração dianteira) com 225 cavalos de potência, acoplado ao transbordo com capacidade de 20 toneladas, em velocidade de deslocamento constante, semelhante a da colhedora.
Os tratamentos empregados, de forma inteiramente casualizada, corresponderam a combinações de duas velocidades da colhedora $\left(\mathrm{km} \mathrm{h}^{-1}\right)$ e duas velocidades de rotação do extrator primário (Rotações por minuto - rpm), além das velocidades habitualmente utilizadas pela usina, como testemunha (Tabela 1). Durante a colheita as velocidades foram constantes, não havendo oscilações que pudessem interferir na confiabilidade dos dados obtidos.

Tabela 1. Descrição dos tratamentos de velocidades de deslocamento da colhedora (VDC) e de rotação do extrator primário (VREP). Fazenda Vista Alegre, Pacaembu, São Paulo

\begin{tabular}{ccc}
\hline Tratamentos & VDC $\left({\left.\mathrm{Km} \cdot \mathrm{h}^{-1}\right)}^{-}\right.$ & VREP (RPM) \\
\hline T1 & 5 & 950 \\
T2 & 3 & 1200 \\
T3 & 6 & 1200 \\
T4 & 3 & 700 \\
T5 & 6 & 700
\end{tabular}

${ }^{1}$ Testemunha com as velocidades comumente utilizadas pela usina.

O levantamento das perdas foi realizado em áreas demarcadas, com 5,6 $\mathrm{m}$ de largura (quatro fileiras) por 5,0 m de comprimento, totalizando $28 \mathrm{~m}^{2}$. As parcelas foram escolhidas aleatoriamente, sendo retiradas dez amostras para cada tratamento avaliado. Nessas áreas, as perdas foram coletadas e classificadas de acordo com Reis (2009) em: a) Tocos (t ha ${ }^{-1}$ ): fração do colmo cortada acima da superfície do solo, presa às raízes não arrancadas, com comprimento menor ou igual a $0,20 \mathrm{~m}$; b) Despontes $\left(\mathrm{t} \mathrm{ha}^{-1}\right)$ : fração de colmo deixada no solo e agregada ao ponteiro. A retirada de cana ponta foi efetuada quebrando-se manualmente o colmo, no ponto de menor resistência; c) Toletes ( $\mathrm{t} \mathrm{ha}{ }^{-1}$ ): fração do colmo com o corte característico do facão picador ou do corte de base, em ambas as extremidades; d) Pedaços $\left(t_{\text {ha }}{ }^{-1}\right)$ : todas as variações visíveis de colmos sem as características que definam tocos, colmos inteiros, rebolos, lascas e cana ponta e que, portanto, não se encaixam em nenhuma das definições anteriormente citadas; e) Canas inteiras $\left(\mathrm{t} \mathrm{ha}^{-1}\right)$ : considerando fração de cana com tamanho igual ou superior a 2/3 do comprimento médio estimado dos colmos do local. Esse colmo pode ou não estar preso ao solo, pelas raízes; f) Estilhaços (t ha ${ }^{-1}$ ): fragmentos de cana dilacerados; g) Perdas totais ( $\mathrm{t} \mathrm{ha}^{-1}$ ): Obtida pela soma de todas as perdas anteriormente citadas e h) \% de perdas: obtida a partir da transformação dos resultados de Perdas Totais em dados percentuais, relativos à produtividade estimada do canavial $\left(75 \mathrm{t} \mathrm{ha}^{-1}\right)$.

Obtidos os valores de porcentagem de perdas totais, os mesmos foram comparados de acordo com a tabela proposta pelo Centro de Tecnologia Canavieira (CTC) (BENEDINI et. al., 2009) (Tabela 2).

Tabela 2. Classificação das perdas segundo o Centro de Tecnologia Canavieira (CTC)

\begin{tabular}{lc}
\hline Nível de Perdas & Percentual de Perdas (\%) \\
\hline Perdas Baixas & $<2,5 \%$ \\
Perdas Médias & $2,5 \%$ a $4,5 \%$ \\
Perdas Altas & $>4,5 \%$ \\
\hline
\end{tabular}

Fonte: Benedini et. al. (2009) 
Os resultados obtidos foram submetidos à análise de variância e ao teste $\mathrm{F} e$ as médias comparadas pelo teste de Tukey em nível de $5 \%$ de probabilidade, através do software estatístico SISVAR (FERREIRA, 2011).

\section{Resultados e Discussão}

Foram observadas respostas significativas $(p \leq 0,05)$ para as perdas nas formas de estilhaços, toletes, pedaços e tocos em relação aos tratamentos avaliados (Tabela 3). Para as perdas na forma de estilhaços, a combinação das velocidades de $6 \mathrm{~km} \mathrm{~h}^{-1}$ e 1200 $\mathrm{rpm}$ proporcionou o maior valor de perdas, entretanto, este valor não diferiu estatisticamente do tratamento composto pela combinação das velocidades de $3 \mathrm{~km} \mathrm{~h}^{-1}$ e 1200 rpm para a variável em questão, enquanto que o tratamento com as mesmas velocidades de deslocamento da colhedora em combinação com a velocidade de $700 \mathrm{rpm}$ de rotação do extrator primário apresentaram os menores valores (Tabela 3).

Em relação as perdas na forma de toletes, o tratamento representado pela combinação das velocidades de $5 \mathrm{~km} \mathrm{~h}^{-1}$ e 950 rpm proporcionou o maior valor de perdas, enquanto que o tratamento com as velocidades de $6 \mathrm{~km} \mathrm{~h}^{-1}$ e $700 \mathrm{rpm}$ apresentou a menor média estimada (Tabela 3 ).

Já para as perdas na forma de pedaços, a combinação das velocidades de $5 \mathrm{~km} \mathrm{~h}^{-1}$ e 950 rpm proporcionou o maior valor médio de perdas enquanto a combinação de $3 \mathrm{~km} \mathrm{~h}^{-1}$ e $700 \mathrm{rpm}$ apresentou o menor valor. Quanto as perdas na forma de toco, as velocidades combinadas de 3 $\mathrm{km} \mathrm{h}^{-1}$ e $700 \mathrm{rpm}$ proporcionaram os valores mais elevados enquanto que a combinação de $3 \mathrm{~km} \mathrm{~h}^{-1}$ e $1200 \mathrm{rpm}$ apresentou a menor quantidade da referida perda.

Tabela 3. Análise de variância e valores médios para Estilhaços (EST) $\left(t \mathrm{ha}^{-1}\right)$, Toletes (TOL) $\left(t \mathrm{ha}^{-1}\right)$, Pedaços (PED) ( $t$ ha ${ }^{-1}$ ) e Tocos (TOC) ( $t$ ha $^{-1}$ ) em função da combinação de diferentes velocidades de locomoção da colhedora $\left(\mathrm{Km} \mathrm{h}^{-1}\right)$ e de rotação do extrator primário $(\mathrm{rpm})$ na colheita mecanizada de cana-de-açúcar de primeiro corte. Fazenda Vista Alegre, Pacaembu, São Paulo

\begin{tabular}{ccccc}
\hline Tratamentos & EST & TOL & PED & TOC \\
\hline $3 \mathrm{~km} \cdot \mathrm{h}^{-1}-1200 \mathrm{rpm}$ & $0.30 \mathrm{ab}$ & $0.52 \mathrm{~d}$ & $0.15 \mathrm{c}$ & $0.07 \mathrm{e}$ \\
$6 \mathrm{~km} \cdot \mathrm{h}^{-1}-1200 \mathrm{rpm}$ & $0.32 \mathrm{a}$ & $0.54 \mathrm{c}$ & $0.38 \mathrm{~b}$ & $0.21 \mathrm{~b}$ \\
$6 \mathrm{~km} \cdot \mathrm{h}^{-1}-700 \mathrm{rpm}$ & $0.08 \mathrm{~d}$ & $0.49 \mathrm{e}$ & $0.15 \mathrm{c}$ & $0.11 \mathrm{~d}$ \\
$3 \mathrm{~km} \cdot \mathrm{h}^{-1}-700 \mathrm{rpm}$ & $0.16 \mathrm{~cd}$ & $0.64 \mathrm{~b}$ & $0.01 \mathrm{~d}$ & $0.22 \mathrm{a}$ \\
$5 \mathrm{~km} \cdot \mathrm{h}^{-1}-950 \mathrm{rpm}$ & $0.23 \mathrm{bc}$ & $0.67 \mathrm{a}$ & $0.68 \mathrm{a}$ & $0.15 \mathrm{c}$ \\
\hline DMS & 0.08 & 0.01 & 0.01 & 0.01 \\
$\mathbf{F}$ & $0.000^{* *}$ & $0.000^{* *}$ & $0.000^{* *}$ & $0.000^{* *}$ \\
C.V(\%) & 17.07 & 1.13 & 2.15 & 3.96 \\
\hline
\end{tabular}

Médias seguidas de mesma letra nas colunas não diferem entre si pelo teste de Tukey no nível de significância de $p>0,05$.

Os resultados supracitados para as perdas na forma de estilhaços evidenciam que tal variável resposta tende a ser influenciada principalmente pela velocidade de rotação do extrator primário. Assim, a tendência obtida corrobora com o exposto por Neves et. al., (2004), que afirmam que as perdas na forma de estilhaços de cana geralmente ocorrem devido à influência da velocidade de rotação do exaustor primário onde, segundo os autores, à medida que se aumenta a rotação do exaustor primário, os toletes passam a ser sugados junto com a palha e a terra, sendo lançados ao campo; ao passar pelos exaustores, os mesmos colidem com as pás do exaustor, sendo dilacerados em lascas (estilhaços) e pedaços, contribuindo para o aumento das perdas.

Segundo Moraes e Neves (1997), a possibilidade de haver maiores perdas na forma de toletes aumenta na medida em que se eleva a eficiência de limpeza dos extratores, ou seja, a velocidade de rotação dos mesmos. A alta velocidade do fluxo de ar, geralmente do extrator primário, causa a sucção dos toletes de cana-deaçúcar que, posteriormente, acabam sendo lançados no campo. Entretanto o presente 
estudo obteve resultados em que as perdas na forma de toletes variaram de forma não proporcional à diferença entre os tratamentos. Salvi (2006) relata ainda que o aumento da perda de tolete pode estar relacionado a diversos outros fatores, como falha de sincronismo entre colhedora e o transbordo; desgaste do assoalho do elevador; flap do elevador danificado ou desajustado; cargas excessivas e sobrecarga no bojo da colhedora.

Carvalho (2009) analisando as perdas visíveis de cana-de-açúcar no campo observou que os índices de perdas na forma de pedaços não foram influenciados pelo aumento da velocidade de deslocamento da colhedora. Entretanto, Santos Neto (2013), trabalhando com combinações de três diferentes velocidades de locomoção da colhedora, três diferentes velocidades de rotação do extrator primário e duas diferentes regulagens do picador, obteve resultados onde o tratamento que se superou em relação aos demais foi de $8 \mathrm{~km} \mathrm{~h}^{-1}$ com $1100 \mathrm{rpm}$, e o tratamento que se apresentou menos eficiente foi da combinação das velocidades de 6 $\mathrm{km} \mathrm{h}^{-1}$ e $1100 \mathrm{rpm}$, evidenciando que tal perda, até certo ponto, ocorre com a redução da velocidade de locomoção da colhedora.

Para as perdas do tipo toco, diferentemente do que foi observado no presente estudo, Segato e Daher (2011) encontraram maiores valores de perda do tipo toco a partir da velocidade de $8 \mathrm{~km} \mathrm{~h}^{-1}$, maior velocidade de locomoção da colhedora empregada em seu experimento. Entretanto, autores como Silva et al. (2015), atribuem perdas do tipo toco a variedade de cana utilizada, assim, os autores não observaram influência da velocidade de deslocamento da colhedora sobre a variável resposta.

Em relação às perdas nas formas de despontes e cana inteira, não houve influencia dos tratamentos $(p>0,05)$, tendo em vista que, para todos os tratamentos, não houveram tais perdas (Tabela 4). Para as perdas totais a velocidade de locomoção de $3 \mathrm{~km} \mathrm{~h}^{-1}$ combinada com $1200 \mathrm{rpm}$ do extrator primário proporcionou os melhores resultados, com o menor valor médio de perdas, enquanto que a combinação de $6 \mathrm{~km} \mathrm{~h}^{-1}$ e $1200 \mathrm{rpm}$, tal como $5 \mathrm{~km} \mathrm{~h}^{-1}$ e $950 \mathrm{rpm}$ proporcionaram os maiores valores para a referida perda (Tabela 4). Já para os valores observados para porcentagem de perda, o maior valor foi proporcionado pela combinação das velocidades de $5 \mathrm{~km} \mathrm{~h}^{-1}$ e $950 \mathrm{rpm}$, enquanto que a combinação das velocidades de $6 \mathrm{~km} \mathrm{~h}^{-1}$ e 700 rpm proporcionou a menor porcentagem de perdas totais (Tabela 4).

Tabela 4. Análise de variância e valores médios para Despontes (DES) $\left(\mathrm{t}\right.$ ha $\left.{ }^{-1}\right)$, Canas Inteiras $(\mathrm{Cl})\left(\mathrm{t} \mathrm{ha}{ }^{-1}\right)$, Perdas Totais (PT) $\left(t h^{-1}\right.$ ) e Porcentagem de Perdas (P \%) em função da combinação de diferentes velocidades de locomoção da colhedora $\left(\mathrm{Km} \mathrm{h}^{-1}\right)$ e de rotação do extrator primário (rpm) na colheita mecanizada de cana-de-açúcar de primeiro corte. Fazenda Vista Alegre, Pacaembu, São Paulo

\begin{tabular}{ccccc}
\hline Tratamentos & DES & Cl & PT & P \% \\
\hline $3 \mathrm{~km} \cdot \mathrm{h}^{-1}-1200 \mathrm{rpm}$ & $0 \mathrm{a}$ & $0 \mathrm{a}$ & $0.77 \mathrm{c}$ & $1.38 \mathrm{~d}$ \\
$6 \mathrm{~km} \cdot \mathrm{h}^{-1}-1200 \mathrm{rpm}$ & $0 \mathrm{a}$ & $0 \mathrm{a}$ & $1.44 \mathrm{a}$ & $1.92 \mathrm{~b}$ \\
$6 \mathrm{~km} \cdot \mathrm{h}^{-1}-700 \mathrm{rpm}$ & $0 \mathrm{a}$ & $0 \mathrm{a}$ & $0.84 \mathrm{bc}$ & $1.12 \mathrm{e}$ \\
$3 \mathrm{~km} \cdot \mathrm{h}^{-1}-700 \mathrm{rpm}$ & $0 \mathrm{a}$ & $0 \mathrm{a}$ & $1.35 \mathrm{ab}$ & $1.80 \mathrm{c}$ \\
$5 \mathrm{~km} \cdot \mathrm{h}^{-1}-950 \mathrm{rpm}$ & $0 \mathrm{a}$ & $0 \mathrm{a}$ & $1.73 \mathrm{a}$ & $2.30 \mathrm{a}$ \\
\hline DMS & --- & --- & 0.51 & 0.03 \\
F & --- & --- & $0.000^{* *}$ & $0.000^{* *}$ \\
C.V(\%) & --- & -- & 18.73 & 0.88 \\
\hline
\end{tabular}

Médias seguidas de mesma letra nas colunas não diferem entre si pelo teste de Tukey no nível de significância de $\mathrm{p}>0,05$.

A não ocorrência de perdas na forma de cana inteira pode ser explicada pelo fato de tais perdas se relacionarem diretamente com a uniformidade do terreno e a forma que o condutor da colhedora a manuseia, onde as perdas ocorrem geralmente quando o condutor comete erros na linha de colheita. Já as perdas na forma de desponte podem ser associadas ao despontador da colhedora desligado ou danificado e ao acamamento da cultura (SALVI, 
2006). Nenhuma das situações mencionadas como agravantes de tais perdas ocorreram durante a condução presente trabalho.

Os resultados apresentados para as perdas totais demonstram que a redução da velocidade de deslocamento da colhedora tende a proporcionar menores valores de perdas totais na colheita. Em contra partida, Neves et al. (2006) afirmaram que os altos índices de perdas de matéria-prima ou a baixa eficiência de limpeza não estão associadas diretamente à velocidade de deslocamento da colhedora, mas ao gradiente do fluxo de ar na câmara de limpeza, que apresenta grande variação na velocidade do ar, interferindo na capacidade de limpeza da colhedora e, por consequência, nas perdas totais, visíveis e invisíveis.

Em relação aos dados de porcentagem de perdas, os valores obtidos foram inferiores aos encontrados por Ripoli et al. (1999), que obtiveram perdas totais de 6,91 e $9,89 \%$ utilizando velocidades de deslocamento da colhedora de 3,0 e 5,0 $\mathrm{km} \mathrm{h}^{-1}$, respectivamente e Romero et al. (1993) de 10 e 13\%, apresentando entretanto, valores superiores àqueles obtidos por Schmidt Junior (2011), de 0,29 a 0,82\% e semelhantes aos valores encontrados por Belardo (2010) entre 1,5 e $3,2 \%$.

Os dados percentuais de perda de matéria prima para todos os tratamentos foram caracterizados como baixos de acordo com o proposto pelo Centro de Tecnologia Canavieira (CTC), que classifica assim as perdas inferiores a 2,5\% (BENEDINI et al., 2009).

\section{Conclusões}

Nas condições de campo apresentadas, o aumento da velocidade de locomoção da colhedora e redução na velocidade da rotação do extrator primário elevam as perdas de cana-de-açúcar. A combinação da velocidade de $3 \mathrm{~km} \mathrm{~h}^{-1}$ com rotação do extrator de $1200 \mathrm{rpm}$ é a mais indicada para a colheita mecanizada da variedade RB96 - 5902.

\section{Referências}

ANTUNES, F. A. F.; CHANDEL, A. K.; BRUMANO, L. P.; TERÁN HILARES, R.; PERES, G. F. D.; AYABE, L. E. S.; SORATO, V. S.; SANTOS, J. R.; SANTOS, J. C.; DA SILVA, S. S. A Novel process intensification strategy for second-generation ethanol production from sugarcane bagasse in fluidized bed reactor. Renewable Energy, v.1, n.1, p.1-8,
2017.

https://doi.org/10.1016/.j.renene.2017.06.004

APOLINÁRIO, G. A.; CORREA, P. A.; CAVALINI, F. C.; GRANATO, P. R.; FERRARI, M. C. Perdas na colheita mecanizada da cana-de-açúcar na usina vista alegre - Itapetininga (SP). Revista Perspectiva em Educação, Gestão \& Tecnologia, v.6, n.12, p.1-7. 2017.

BELARDO, G. C. Avaliação de desempenho efetivo de três colhedoras em cana-de-açúcar (Saccharum spp) sem queima. 2010. 136 p. Dissertação (Mestrado) - Escola Superior de Agricultura "Luiz de Queiroz", Piracicaba, 2010.

BENEDINI, M. S.; BROD, F. P. R.; PERTICARRARI, J. $G$. Perdas e impurezas vegetais e minerais na colheita mecanizada. Boletim técnico. Guariba, 2009. $7 p$.

BRAUNBECK, O.A.; OLIVEIRA, J.T.A. Colheita de cana-de-açúcar com auxílio mecânico. Engenharia Agrícola, 2006.

BURLEIGH. A comparative evaluation of sugarcane harvesting and transport systems for use in the brazilian sugar industry. São Paulo: Copersucar, 1998. 54 p. (Relatório Técnico Copersucar)

CARVALHO, L. S. Desempenho operacional de uma colhedora em cana crua na região da grande dourados - MS. 2009. 36p. Dissertação (Mestrado em Produção Vegetal) - Universidade Federal da Grande Dourados, Dourados, 2009.

CONAB. Acompanhamento da Safra Brasileira: cana-de-açúcar - Safra 2017/18. Quarto levantamento, v.4, n.4. 2018.

COSTA NETO, J. D. A cana em tempo bom. Revista CREA-PR, n.41, p.16-19, 2006.

FERREIRA, D.F. Sisvar: a computer statistical analysis system. Ciência e Agrotecnologia, v.35, n.6, p.1039-1042, 2011. http://dx.doi.org/10.1590/S1413$\underline{70542011000600001}$

MAGALHAES, P. S. G.; BALDO, R. F. G.; CERRI, D. G. P. Sistema de sincronismo entre a colhedora de cana-de-açúcar e o veículo de transbordo. Engenharia Agrícola, v.28, n. 2, p.274-282, 2008. 
http://dx.doi.org/10.1590/S0100-

\section{$\underline{69162008000200008}$}

MORAES, E.E.; NEVES, J.L.M. Colheita de cana crua: avaliação de perdas invisíveis nos sistemas com colhedoras de cana picada. In: SEMINÁRIO DE TECNOLOGIA AGRONÔMICA, 7., 1997, Piracicaba. Anais [...] Piracicaba: COPERSUCAR, 1997.p. 288-297.

NEVES, J. L. M. Avaliação de perdas invisíveis em colhedoras de cana-de-açúcar e alternativas para sua redução. 2003. 223 p. Tese (Doutorado em Engenharia Agrícola) - Faculdade de Engenharia Agrícola, Universidade Estadual de Campinas, Campinas, 2003.

NEVES, J. L. M.; MAGALHÃES, P. S. G.; OTA, W. M. Sistema de monitoramento de perdas visíveis de cana-de-açúcar em colhedora de cana picada. Engenharia Agrícola, Jaboticabal, v.24, n.3, p.764-770, 2004.

http://dx.doi.org/10.1590/S0100-

\section{$\underline{69162004000300030}$}

NEVES, J.L.M.; MAGALHÃES, P.S.G.; MORAES, E.E.; ARAÚJO, F.V.M. Avaliação de perdas invisíveis na colheita mecanizada em dois fluxos de massa de cana-de-açúcar. Engenharia Agrícola, v.26, n.3, p.787-794, 2006. http://dx.doi.org/10.1590/\$0100$\underline{69162006000300016}$

REIS, G. N. Perdas na colheita mecanizada da cana-de-açúcar crua em função do desgaste das facas do corte de base. 2009. 73 p. Tese (Doutorado) - Faculdade de Ciências Agrárias e Veterinárias, Universidade Estadual Paulista. Jaboticabal, 2009.

RIPOLI, T. C.; NERY, M. S.; DE LEÓN, M. J.; PIEDADE, S. M. S. Desempenho operacional de uma colhedora em cana crua em função da velocidade de avanço. Engenharia Agrícola: Engenharia Agrícola, v.19, n. 2, p. 199-207, 1999.

RIPOLI, T.C.C.; NERY, M.S.; MOLINA JUNIOR, W.F.; RIPOLI, M.L.C. Operation and economic performance of a green cane chopped harvester in Brazil. In: ASAE ANNUAL INTERNATIONAL MEETING, 2001, Sacramento. Anais [...]. St. Joseph: ASAE, 2001. (Paper, 011003).
RIPOLI, T. C. C.; RIPOLI, M. L. Biomassa de canade-açúcar: colheita, energia e ambiente. Piracicaba: Barros \& Marques. 302p, 2004.

SALVI, J.V. Qualidade do corte de base de colhedoras de cana-de-açúcar. 2006. 89f. Dissertação (Mestrado). Escola Superior de Agricultura Luiz de Queiroz. Universidade de São Paulo. Piracicaba, 2006.

SANTOS NETO, J. F. Quantificação de perdas de matéria prima no campo para diferentes regulagens de uma colhedora de cana-de-açúcar no município de Arês - RN. 2013. 34p. Monografia (Graduação em Agronomia) - Centro de Ciências Agrárias, Universidade Federal da Paraíba, Areia- PB, 2013.

SEGATO, S.V.; DAHER, F. Perdas visíveis na colheita mecanizada de cana-de-açúcar crua sob velocidades de deslocamento da colhedora. Nucleus, v.8, 2011. p. 315-326. http://dx.doi.org/10.3738/1982.2278.471

SCHMIDT JUNIOR, J. C. Avaliação do desempenho efetivo de colhedora de cana-deaçúcar (saccharum spp.). 2011. 108 p. Dissertação (Mestrado em Máquinas agrícolas) Escola Superior de Agricultura "Luiz de Queiroz", Universidade de São Paulo, Piracicaba, 2011.

SILVA, R.C.; OLIVEIRA, T.C.; FIGUEIREDO, Z.N.; CALDEIRA, D.S.A. Perdas visíveis na colheita mecanizada de cana-de-açúcar. Engenharia na Agricultura, v.23, n.1, p.71-77, 2015.

YOUNGER, J.A. Quality cane and extraneous matter. In: INTERNATIONAL SOCIETY OF SUGARCANE TECHNOLOGISTS CONGRESS, 17., 1980, Manila. Proceedings [...]. Philippines: The Executive Commite of the ISSCT Congress, 1980. v.1, p.885-90. 